\title{
Theory of intrinsic propagation losses in topological edge states of planar photonic crystals
}

\author{
Erik Sauer, ${ }^{1, *}$ Juan Pablo Vasco $\odot,{ }^{1,2, \dagger}$ and Stephen Hughes $\odot^{1, *}$ \\ ${ }^{1}$ Department of Physics, Engineering Physics and Astronomy, Queen's University, Kingston, Ontario K7L 3N6, Canada \\ ${ }^{2}$ Institute of Theoretical Physics, École Polytechnique Fédérale de Lausanne EPFL, CH-1015 Lausanne, Switzerland
}

(Received 26 May 2020; revised 9 September 2020; accepted 21 September 2020; published 20 October 2020)

\begin{abstract}
Using a semianalytic guided-mode expansion technique, we present theory and analysis of intrinsic propagation losses for topological photonic crystal slab waveguide structures with modified honeycomb lattices of circular or triangular holes. Although conventional photonic crystal waveguide structures, such as the W1 waveguide, have been designed to have lossless propagation modes, they are prone to disorder-induced losses and backscattering. Topological structures have been proposed to help mitigate this effect as their photonic edge states may allow for topological protection. However, the intrinsic propagation losses of these structures are not well understood and the concept of the light line can become blurred. For four example topological edge state structures, photonic band diagrams, loss parameters, and electromagnetic fields of the guided modes are computed. Two of these structures, based on armchair edge states, are found to have significant intrinsic losses for modes inside the photonic bandgap, more than $100 \mathrm{~dB} / \mathrm{cm}$, which is comparable to or larger than typical disorder-induced losses using slow-light modes in conventional photonic crystal waveguides, while the other two structures, using the valley Hall effect and inversion symmetry, are found to have a good bandwidth for exploiting lossless propagation modes below the light line (at least in the absence of disorder).
\end{abstract}

DOI: 10.1103/PhysRevResearch.2.043109

\section{INTRODUCTION}

Semiconductor photonic crystals (PCs) are dielectric structures that allow the manipulation of light on the nanoscale, achieved by tailoring the periodicity of the dielectric constant [1-4]. In particular, planar photonic crystal slabs (PCSs) have a two-dimensional in-plane periodicity in their lattice structure, which can be used to realize slow-light modes on semiconductor chips [5]. The PCSs are often introduced with defects within their lattice structures, e.g., to create waveguides [3,4,6-11], which allow the propagation of light in a particular direction, or trap light in cavities [12-23]. The fabrication of these PCSs is made possible through semiconductor growth techniques [2], such as etching [24] and lithography [25].

In terms of understanding extrinsic (i.e., disorder-induced) propagation losses, conventional PCS waveguide structures, such as the W1 waveguide (i.e., a single row of missing holes), have been studied extensively [2,26]. Kuramochi et al. [27] have achieved PCS waveguide losses as low as $5 \mathrm{~dB} / \mathrm{cm}$, and O'Faolain et al. [28] as low as $15 \mathrm{~dB} / \mathrm{cm}$. Variations of the $\mathrm{W} 1$ design can help improve these numbers somewhat in terms of reducing the loss per group index [29,30]. However, in all of these conventional designs, operation near the mode edge (slow light regime) becomes impractical because of significant disorder-induced backscattering [2,26,31-39].

\footnotetext{
*14ers4@queensu.ca

†juan.vasco@epfl.ch

†shughes@queensu.ca
}

Published by the American Physical Society under the terms of the Creative Commons Attribution 4.0 International license. Further distribution of this work must maintain attribution to the author(s) and the published article's title, journal citation, and DOI.
In recent years, it has been proposed that "topological" photonic structures can help mitigate the problem of disorderinduced losses in PCS waveguides, thanks to the special properties of their photonic edge states. These edge states of topological waveguides may allow scatter-free propagation for nanoscale PCs and have applications in quantum technologies due to their strong interactions with quantum emitters [40-47]. Experimentally, electromagnetic modes for these topological edge states have been measured by Barik et al. [48] in 2018, indicating that these topological edge states can function as waveguides, with localized spin control. However, for these PCS geometries, the role of intrinsic out-of-plane losses on the propagating modes is not well understood. Quantifying such radiative losses is essential to properly characterize the topological edge states in PCSs and, ultimately, to improve their performance and understanding.

To accurately model the behavior of light within PCSs, numerical solutions to Maxwell's equations in the full three-dimensional geometry are required [3,49-51]. For this purpose, well-known numerical approaches, such as the finite-difference time-domain (FDTD) method [52] and the plane-wave expansion (PWE) method [53,54], have been commonly employed during the last two decades. FDTD techniques directly solve Maxwell's equations by iterating through time, on a finite spatial grid. The FDTD method is numerically exact (i.e., without approximations), however, it is a brute-force method which can be computationally inefficient $[3,55]$. This computational inefficiency is especially problematic when computing modes above the light line in three dimensions, and lossy waveguide modes can be hard or impossible to resolve with a time-dependent solution. The PWE method, on the other hand, works in the frequency domain rather than in the time domain; PWE solves Maxwell's equations as an eigenvalue problem and is 
significantly more efficient than FDTD. However, a major limitation with PWE is that it assumes periodicity in all spatial directions and can only be accurately used for lossless systems, such as standard PCS waveguides operating below the light line $[2,56]$.

An alternative approach to the brute-force solvers like FDTD is the semianalytical method, originally proposed by Andreani and Gerace, known as the guided-mode expansion (GME) [57] method. In GME, the magnetic field of the PCS is expanded in the basis of the guided mode of the slab's effective waveguide, and the resulting eigenvalue equation is solved numerically. The benefits of the GME method are twofold: (i) it is significantly more computationally efficient than other numerical methods such as FDTD, because the matrix elements of the Maxwell operator become analytical in the guided-mode basis; and (ii) the imaginary part of the eigenvalue, which accounts for the out-of-plane losses, can be obtained by using time-dependent perturbation theory in the low-loss regime. This makes the GME an ideal theoretical tool for numerically solving PCSs when the imaginary part of the mode frequency is much smaller than its real part. Thus, the GME method is an excellent and efficient method of choice to analyze the photonic band structure and intrinsic propagation losses of topological PCS waveguides.

In this work, we study four topological PCS waveguide designs that have been recently presented in the literature. The PCS waveguides are analyzed using the GME approach, where we compute the complex photonic band structure and quantify the intrinsic radiation losses above the light line. We also identify the regions where these out-of-plane losses are minimized and characterize the corresponding intensity profiles of the waveguide modes. The first two structures are based on the designs from Anderson and Subramania [40] and Barik et al. [41], which we show to be intrinsically lossy and form modes inside the photonic bandgap but above the light line, while the latter two structures, from Shalaev et al. [58] and He et al. [59], do have have edge states modes below the light line and are thus more promising in terms of mitigating problems of intrinsic diffraction losses. Importantly, all these structures could be efficiently redesigned using the GME to improve their loss characteristics, though we will not study optimization techniques in this paper.

The layout of the rest of our paper is as follows: Section II introduces the main designs of interest, Sec. III presents the GME theory and methods for computing complex band structure and losses, and Sec. IV presents our main results for the four waveguide designs. Our conclusions are presented in Sec. V. We also include three Appendixes: in Appendix A we provide further details of the intrinsic loss calculations and features; in Appendix B we provide more examples of the various waveguide modes found by the GME; and in Appendix $\mathrm{C}$ we provide further waveguide mode graphs to display the chiral properties of the edge state waveguide modes, which have applications for coupling to quantum emitters with unidirectional control.

\section{DESIGNS FOR TOPOLOGICAL EDGE STATES IN PHOTONIC CRYSTAL WAVEGUIDES}

Figure 1 shows two schematic examples of PCS structures that support topological edge states, using circles or trian-
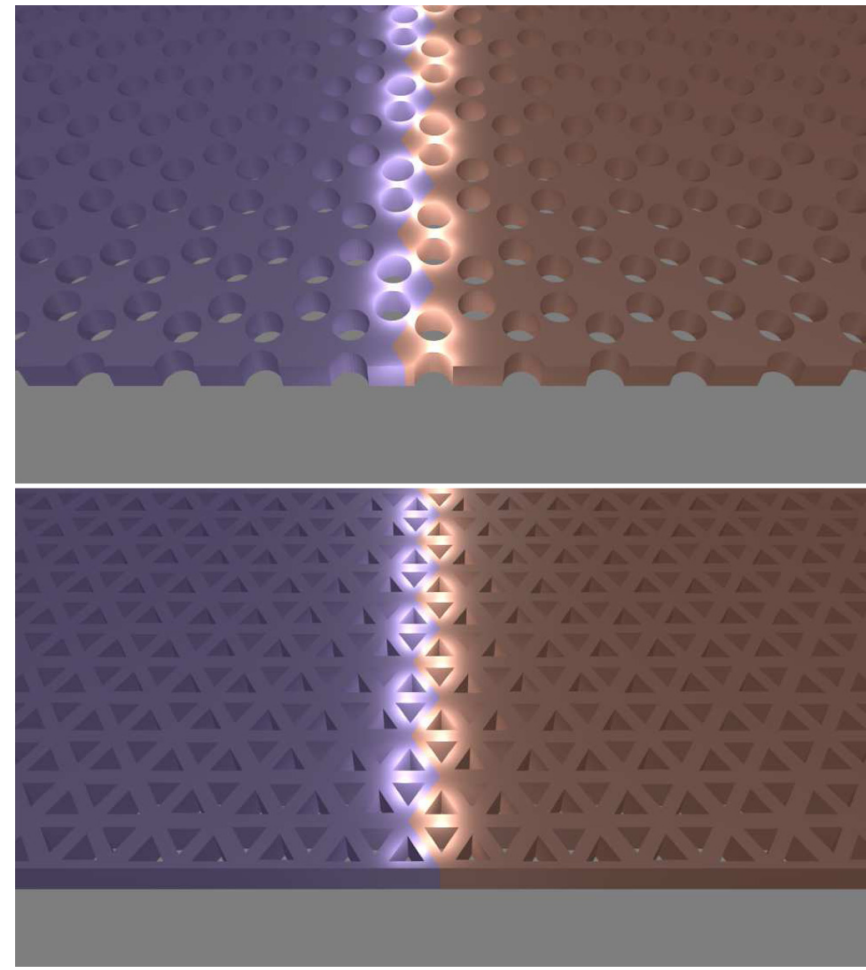

FIG. 1. Schematic three-dimensional models of two example topological PCS structures: one with circular holes and one with triangular holes. An interface, which acts as a waveguide, separates two lattice structures (in this example, with expanded or shrunken honeycomb lattices).

gles on a semiconductor slab (or membrane). The interfaces in these two examples separate a topologically trivial lattice structure with shrunken honeycomb clusters and a topologically nontrivial lattice structure with expanded honeycomb clusters. These two designs, from Refs. [40,41], use standard honeycomb lattices, with an armchair interface separating expanded and shrunken honeycomb clusters. We refer to these structures as armchair edge state designs. Anderson and Subramania [40] have presented theoretical photonic band structure calculations for their design of circular holes, as well as power flow diagrams along the interface. However, propagation losses were not considered. For the design by Barik et al. [41], impressive experimental measurements were also demonstrated by coupling quantum dot emitters to the waveguide modes [48]. Some partial loss calculations for this structure are available in the Supplemental Material to Ref. [48], which are presented in the form of a minimum propagation length using the FDTD technique. Experimentally, a loss length of $22 \mu \mathrm{m}$ was shown for this structure, and they predicted that a loss length of up to $40 \mu \mathrm{m}$ could be achieved with appropriate parameter adjustments. With such a brute-force FDTD approach, the origin of such losses is not so clear; alternative techniques are thus needed not only to highlight the underlying physics, but also to efficiently explore the parameter space for lower loss designs.

Very recent designs from Refs. [58,59] use honeycomb clusters with two alternating hole sizes and instead use inversion symmetry to achieve a waveguide interface. The design 
proposed by Shalaev et al. [58] is introduced as a topological insulator that exhibits the valley Hall effect at telecommunications wavelengths. A similar design by $\mathrm{He}$ et al. [59] also exhibits valley Hall effects and has been experimentally investigated on top of a $\mathrm{SiO}_{2}$ substrate. The design that we investigate below does not consider the substrate for GME calculations, so that it is consistent with the three other designs, since air bridge structures have less overlap with the light line. Impressive experiments have been done with both these structures and low losses have been reported. We refer to these two structures as valley Hall edge state designs.

\section{GUIDED-MODE EXPANSION TECHNIQUE AND PROPAGATION LOSSES}

For linear and nonmagnetic media, one can rewrite Maxwell's equations in the frequency domain, such that a second-order eigenvalue equation in terms of the magnetic field $\boldsymbol{H}(\boldsymbol{r})$ is obtained,

$$
\nabla \times\left[\frac{1}{\epsilon(\boldsymbol{r})} \nabla \times \boldsymbol{H}(\boldsymbol{r})\right]=\left(\frac{\omega}{c}\right)^{2} \boldsymbol{H}(\boldsymbol{r}),
$$

where $\epsilon(\boldsymbol{r})$ is the dielectric constant of the slab. To solve this eigenvalue problem using the GME method, the magnetic field is expanded in an orthonormal set of basis states,

$$
\boldsymbol{H}(\boldsymbol{r})=\sum_{\mu} c_{\mu} \boldsymbol{H}_{\mu}(\boldsymbol{r}),
$$

with the orthonormality condition,

$$
\int_{\text {unit cell }} \boldsymbol{H}_{\mu}^{*}(\boldsymbol{r}) \cdot \boldsymbol{H}_{\nu}(\boldsymbol{r}) d \boldsymbol{r}=\delta_{\mu, \nu} .
$$

Then Eq. (1) is rewritten as a linear eigenvalue problem,

$$
\sum_{\nu} \mathcal{H}_{\mu \nu} c_{\nu}=\frac{\omega^{2}}{c^{2}} c_{\mu}
$$

where the matrix elements $\mathcal{H}_{\mu \nu}$ are defined as

$$
\mathcal{H}_{\mu \nu}=\int \frac{1}{\epsilon(\boldsymbol{r})}\left(\nabla \times \boldsymbol{H}_{\mu}^{*}(\boldsymbol{r})\right) \cdot\left(\nabla \times \boldsymbol{H}_{\nu}(\boldsymbol{r})\right) d \boldsymbol{r} .
$$

To solve for $\mathcal{H}_{\mu \nu}$, the GME method obtains the magnetic field for each Bloch wave vector $\boldsymbol{k}$ as a sum of the guided modes over the reciprocal lattice vectors and the mode index $i$. Therefore, the GME for the magnetic field can be rewritten as

$$
\boldsymbol{H}_{\boldsymbol{k}}(\boldsymbol{r})=\sum_{\boldsymbol{G}, i} c(\boldsymbol{k}+\boldsymbol{G}, i) \boldsymbol{H}_{\boldsymbol{k}+\boldsymbol{G}, i}^{\text {guided }}(\boldsymbol{r}),
$$

where $\boldsymbol{G}$ is a reciprocal lattice vector for the PCS's lattice structure. The analytical solution for the guided mode $H_{\boldsymbol{k}+\boldsymbol{G}, i}^{\text {guided }}(\boldsymbol{r})$ varies depending on the slab's layer and whether the mode is transverse electric (TE) or transverse magnetic (TM) [57]. Note that the matrix elements $\mathcal{H}_{\mu \nu}$ in Eq. (4) depend on the Fourier transform of the inverse dielectric function in each slab layer $j=\{1,2,3\}$, through

$$
\eta_{j}\left(\boldsymbol{G}, \boldsymbol{G}^{\prime}\right)=\frac{1}{A} \int_{\text {cell }} \epsilon_{j}(\boldsymbol{\rho})^{-1} e^{i\left(\boldsymbol{G}^{\prime}-\boldsymbol{G}\right) \cdot \boldsymbol{\rho}} d \boldsymbol{\rho},
$$

where $A$ is the unit cell area, $\rho=(x, y)$, and $j$ represents one of the slab's three layers: the lower cladding, the core, and the upper cladding. However, from a numerical perspective, it is much more convenient to calculate the matrix elements of the dielectric function directly as [57]

$$
\epsilon_{j}\left(\boldsymbol{G}, \boldsymbol{G}^{\prime}\right)=\frac{1}{A} \int_{\text {cell }} \epsilon_{j}(\boldsymbol{\rho}) e^{i\left(\boldsymbol{G}^{\prime}-\boldsymbol{G}\right) \cdot \boldsymbol{\rho}} d \boldsymbol{\rho}
$$

and use numerical matrix inversion to find $\eta_{j}\left(\boldsymbol{G}, \boldsymbol{G}^{\prime}\right)=$ $\epsilon_{j}^{-1}\left(\boldsymbol{G}, \boldsymbol{G}^{\prime}\right)$. This is the approach that we take.

The guided-mode basis is computed in an effective homogeneous slab whose dielectric constant is usually taken as the spatial average of $\epsilon_{j}(\rho)$ :

$$
\bar{\epsilon}_{j}=\frac{1}{A} \int_{\text {cell }} \epsilon_{j}(\rho) d \rho .
$$

Once the magnetic field is obtained from Eq. (6), the electric field is obtained by

$$
\boldsymbol{E}_{\boldsymbol{k}}(\boldsymbol{r})=\frac{i c}{\omega \epsilon(\boldsymbol{r})} \times \boldsymbol{H}_{k}(\boldsymbol{r}),
$$

where

$$
\int_{\text {unit cell }} \epsilon(\boldsymbol{r}) \boldsymbol{E}_{\boldsymbol{k}}^{*}(\boldsymbol{r}) \cdot \boldsymbol{E}_{\boldsymbol{k}^{\prime}}(\boldsymbol{r}) d \boldsymbol{r}=\delta_{k, k^{\prime}}
$$

Although performing the GME in this way is accurate for photonic modes below the light line, it does not directly obtain out-of-plane (intrinsic) losses. However, since such losses are small, one can can estimate these losses using perturbation theory. Specifically, when a photonic mode escapes the slab's core into the claddings, it couples to lossy radiation modes and falls above the light line. The mode becomes quasiguided and is now subject to intrinsic losses, which can be accurately computed from the imaginary part of the eigenfrequency, $\operatorname{Im}(\omega)$. Similarly to Fermi's golden rule from quantum mechanics, these losses can be computed by second-order time-dependent perturbation theory [57], from the photonic golden rule:

$$
-\operatorname{Im}\left(\frac{\omega_{k}^{2}}{c^{2}}\right)=\pi \sum_{\boldsymbol{G}^{\prime}} \sum_{\lambda} \sum_{j=1,3}\left|\mathcal{H}_{\boldsymbol{k}, \mathrm{rad}}\right|^{2} \rho_{j}\left(\boldsymbol{k}+\boldsymbol{G}^{\prime} ; \frac{\omega_{k}^{2}}{c^{2}}\right),
$$

where $\lambda$ represents either a TE or a TM mode, and the matrix element between a guided and lossy radiation mode is given by

$$
\mathcal{H}_{\boldsymbol{k}, \mathrm{rad}}=\int \frac{1}{\epsilon(\boldsymbol{r})}\left(\boldsymbol{\nabla} \times \boldsymbol{H}_{\boldsymbol{k}}^{*}(\boldsymbol{r})\right) \cdot\left(\boldsymbol{\nabla} \times \boldsymbol{H}_{\boldsymbol{k}+\boldsymbol{G}^{\prime}, \lambda, j}^{\mathrm{rad}}(\boldsymbol{r})\right) d \boldsymbol{r},
$$

and $\rho_{j}$ is the one-dimensional photonic density of states for a given wave vector $\boldsymbol{g}=\boldsymbol{k}+\boldsymbol{G}$ in layer $\boldsymbol{j}$,

$$
\rho_{j}\left(g ; \frac{\omega^{2}}{c^{2}}\right)=\frac{\bar{\epsilon}_{j}^{-1 / 2} c}{4 \pi} \frac{\theta\left(\omega^{2}-\frac{c^{2} g^{2}}{\bar{\epsilon}_{j}}\right)^{-1 / 2}}{\left(\omega^{2}-\frac{c^{2} g^{2}}{\bar{\epsilon}_{j}}\right)^{-1 / 2}},
$$

with $\theta$ representing the Heaviside step function. Similarly to those for the guided modes, the analytical definitions for the radiation modes $H_{\boldsymbol{k}+\boldsymbol{G}^{\prime}, \lambda, j}^{\mathrm{rad}}(\boldsymbol{r})$ depend on the slab's layer and polarization [57]. 
The imaginary part of the frequency is then easily obtained from

$$
\operatorname{Im}\left(\omega_{k}\right)=\frac{\operatorname{Im}\left(\omega_{k}^{2}\right)}{2 \operatorname{Re}\left(\omega_{k}\right)} .
$$

Subsequently, the power loss coefficient, $\alpha$, is obtained from

$$
\alpha=2 \frac{\operatorname{Im}\left(\omega_{k}\right)}{\left|v_{g}\right|},
$$

where $v_{g}=d \omega / d k$ is the mode group velocity. Another useful parameter for connecting to experiments is power loss in decibels $(\mathrm{dB})$, obtained from $\operatorname{Loss}_{\mathrm{dB}}=4.34 \alpha$.

It is worth commenting on the expected accuracy of the GME loss calculations. As expected from any perturbative approach, the GME method is an approximate one and its predictions are accurate as long as the underlying assumptions are fulfilled. For the photonic dispersion, structures with high-contrast refractive indices are required in order to make a reliable description of PCS eigenmodes with the vertically confined guided-mode basis, while for the out-of-plane losses, the imaginary part of the frequency, computed with Eqs. (12) and (15), must be much smaller than its real part. These two conditions are perfectly satisfied in our cases of interest, where air-bridge high-index slabs are considered and the ratio $\operatorname{Im}(\omega) / \operatorname{Re}(\omega)$ is of the order of $10^{-4}$ for the largest losses computed below. Nevertheless, structures with weakly confined modes in the vertical direction and with strong radiative losses components are likely not suitable for the GME and could require first-principles simulations, such as FDTD; such modes are not considered in this work, and the GME is a far more efficient method to obtain the propagation losses of interest. We also stress that the GME method presents a full vector and three-dimensional solution of Maxwell's equations for a wide range of PCS structures.

\section{NUMERICAL RESULTS FOR THE COMPLEX BAND STRUCTURES AND PROPAGATION LOSSES}

In this section, we apply the GME to the four topological PCS structures described in Sec. II, which we refer to as designs A [40], B [41], C [58], and D [59], respectively. For each of the four designs, photonic band diagrams in the $k_{x}$ direction are computed, along with the nominal light line, and the topological edge states are indicated. A top-down view of each lattice structure's supercell is shown, with the propagation being in the $x$ direction in each case. Since these band diagrams are symmetric about $k_{x}=0$, only the results for $k_{x} \geqslant 0$ are shown. Zoom-ins of these guided bands of interest are shown, along with their intrinsic losses presented in terms of the group index, $n_{g}=\left|c / v_{g}\right|$, where $v_{g}$ is the group velocity, and the loss coefficient, $\alpha$. Finally, mode profiles of the (normalized) electric displacement field, $\boldsymbol{D} \equiv \epsilon \boldsymbol{E}$, are presented, which provide a visual representation of how well the modes remain confined along the waveguides for their respective topological structure.

Our computational implementation of the GME for the structures below was done in MATLAB. To obtain all necessary results, the number of $\boldsymbol{k}$ points and the number of basis states were chosen for the dispersion calculations. These numbers are dependent on the PCS structure. For design A, a total

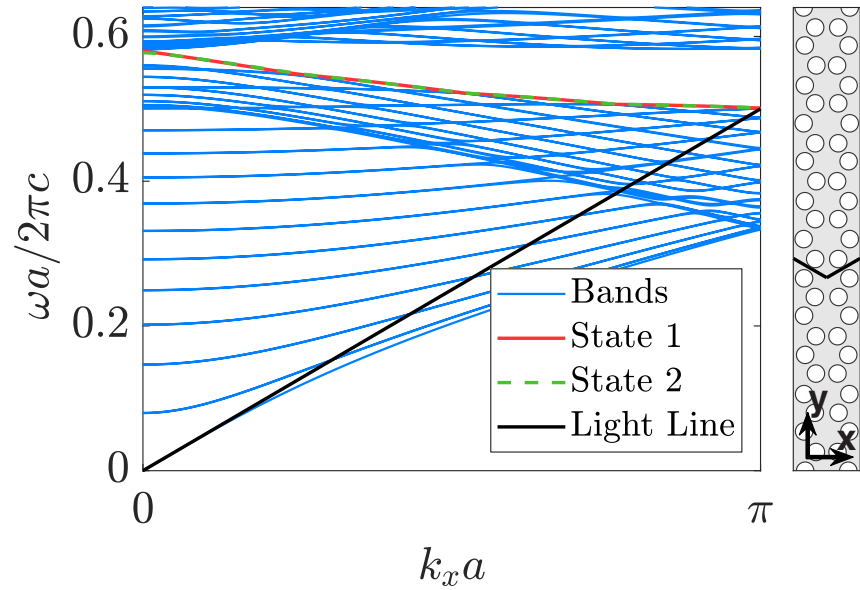

FIG. 2. Dispersion of the lossy armchair interface structure of circular holes, design A [40]. Propagation is along $x$.

of 81 basis states and $1002 k$ points were used. For design B, 144 basis states were computed, with a total of $3002 \boldsymbol{k}$ points. Both design $\mathrm{C}$ and design $\mathrm{D}$ used 60 basis states, with $\mathrm{C}$ using $1502 \boldsymbol{k}$ points and D using $1002 \boldsymbol{k}$ points. For designs A, B, and $\mathrm{D}$, the normalized cutoff in reciprocal lattice vectors, $\boldsymbol{G} a$, was set to 30 , whereas it was set to 40 for design C. These were all checked carefully to ensure numerical convergence.

A. Armchair interfaces of circular holes by Anderson and Subramania [40] and of triangular holes by Barik et al. [41]

We first show results for the PCS structure of the armchair interface of circular holes, introduced by Anderson and Subramania [40]. The GME computations use a slab dielectric constant of $\epsilon_{s}=11.5$, a slab thickness of $d=0.25 a$, a hole radius of $r=0.13 a$, and a lattice constant of $a=870 \mathrm{~nm}$. Assuming that the radius of each honeycomb cluster is $R$ and the lattice constant is $a$, the topologically nontrivial side has expanded honeycomb clusters with $R_{\exp }=a / 2.9$ and the topologically trivial side has shrunken honeycomb clusters with $R_{\mathrm{shr}}=a / 3.1$.

The lattice structure and photonic band diagram for this topological structure is shown in Fig. 2. One might expect the topological edge states in this case to be below the light line, however the GME identifies them to be above the light line, which results in nonzero losses.

It is also important to note that the propagation losses here do not arise from backscattering but, rather, from radiation leaking out vertically while the mode is propagating along the waveguide. However, we are not considering any structural disorder.

Figure 3(a) shows a zoomed-in region of interest of the band structure, highlighting two edge state modes, labeled state 1 and state 2 . The corresponding group index and propagation losses are shown in Fig. 3(b). We identify a point of minimum loss for state 2 , at $k_{x} a=0.091$. The minimum loss coefficient is $\alpha_{\min }=1 / 97 a$, which yields a maximum loss length of $L_{\alpha}=97 a$.

With the lattice constant of $a=870 \mathrm{~nm}$, the minimum losses in this structure were found to be equal to $510 \mathrm{~dB} / \mathrm{cm}$. This quantity is significantly larger than typical 
(a)

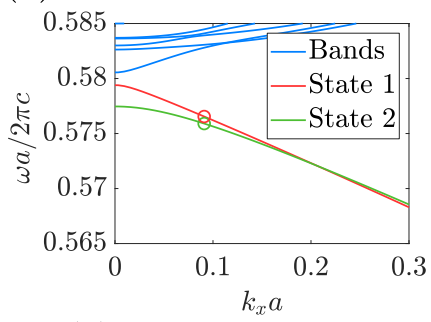

(c)

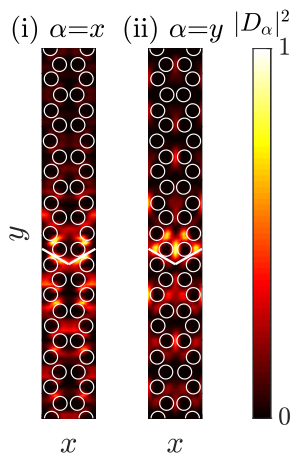

(b)

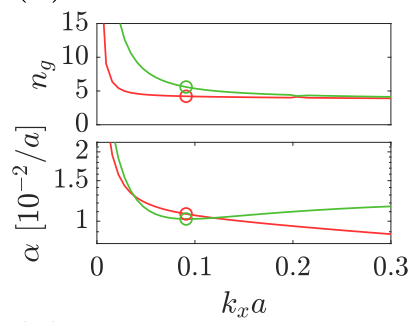

(d)

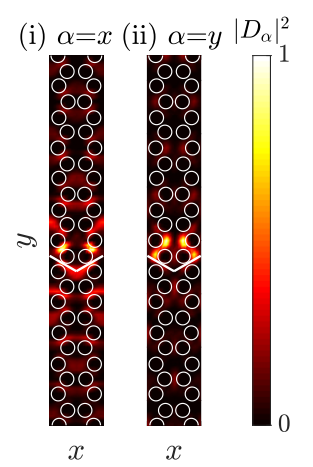

FIG. 3. (a) Zoom-in to design A's guided bands of interest, labeled state 1 and state 2 . Points of interest, which are found above the light line (but within the photonic bandgap), provide the minimum loss coefficient for state 2 (and therefore the maximum loss length) and are indicated by circles. (b) The group index (top), $n_{g}=\left|c / v_{g}\right|$, and loss coefficient (bottom), $\alpha$, of state 1 and state 2 , shown on a logarithmic scale. The two points of interest are represented by circles. (c) Electric displacement field mode profiles of the $x$ and $y$ components of state 1 at the point of interest. (d) Electric displacement field mode profiles of state 2 .

disorder-induced losses of conventional PCS waveguide modes, which are around $5-30 \mathrm{~dB} / \mathrm{cm}$ for the fast-light regime and around $100-1000 \mathrm{~dB} / \mathrm{cm}$ for the slow-light regime $\left(n_{g} \approx 100\right)$ [2,26-28,30,33]. For thin samples, extrinsic (disorder-induced) losses scale inversely with the group index squared (as a general approximation) [2,32]. Intrinsic losses of $\mathrm{W} 1$ waveguides (i.e., above the light line) have also been measured to be around $400 \mathrm{~dB} / \mathrm{cm}$ [27], which is close to the values of the topological edge states here.

The $x$ and $y$ components of the Bloch-mode displacement fields $\boldsymbol{D}$ at $z=0$ (i.e., in the vertical center of the slab) are shown in Figs. 3(c) and 3(d). These modes, shown for state 1 and state 2 from Fig. 3(a), are taken at the points of minimum loss. As expected, the modes remain mostly confined along the interface, however, they are still relatively lossy and confinement seems to be somewhat poor for these edge states.

We also studied the results for a similar PCS structure using the zigzag interface of circular holes [40], and found similar results (not shown). The loss length was found to be equal to $L_{\alpha}=166 a$, which is equivalent to propagation losses of $173.5 \mathrm{~dB} / \mathrm{cm}$, given a waveguide lattice constant of $a \sqrt{3}$. The smaller losses in this structure compared to its armchair counterpart partly stem also from its larger effective mode volume, $V_{\text {eff }}=1 / \max \left[\epsilon(\boldsymbol{r})|\boldsymbol{E}(\boldsymbol{r})|^{2}\right]$ [60], which influences the photonic golden rule calculations. Note that this is an effective mode volume per unit cell [60]. As is clear from Eqs. (12) and (13), losses are also influenced by complex interference

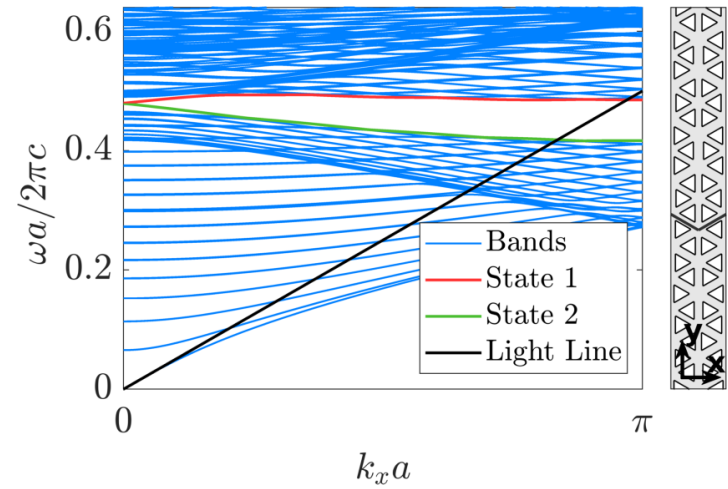

FIG. 4. Dispersion of the lossy armchair interface structure of triangular holes, design B [41]. Propagation is along $x$.

effects between the confined Bloch mode and the radiation modes. Although the zigzag structure has somewhat lower losses, by approximately a factor of 2 , the modes are still above the light line and have significant losses throughout all of $k$ space.

We next consider the PCS structure proposed by Barik et al. [41], which was also demonstrated experimentally by coupling spin-charged quantum dots [48]. The GME computation uses the following parameters: a slab dielectric constant of $\epsilon_{s}=12.11$, a slab thickness of $d=160 a / 445$, a length of one side of the equilateral triangular hole of $L=140 a / 445$, and a lattice constant of $a=445 \mathrm{~nm}$. In this case, the topologically nontrivial side has expanded honeycomb clusters with $R_{\exp }=1.05 a / 3$ and the topologically trivial side has shrunken honeycomb clusters with $R_{\mathrm{shr}}=0.94 a / 3$.

Figure 4 displays the photonic band diagram for this topological structure and its lattice design. Figure 5(a) shows a zoom-in of the region of interest, with two identified edge state modes. Once again, we find that these modes are well above the light line when inside the photonic bandgap, though some of the modes fall below the light line when below the photonic bandgap. Specifically, state 1 resides above the light line for $\left|k_{x} a\right|<3.049$, whereas state 2 resides above the light line for $\left|k_{x} a\right|<2.628$. Within the photonic bandgap region, minimum losses occur at $k_{x} a=0.018$ (for state 2), and the corresponding group index and loss values are shown in Fig. 5(b). Here, the loss coefficient achieved at the point of minimum loss is equal to $\alpha_{\min }=1 / 79 a$, which corresponds to a maximum loss length of $L_{\alpha}=79 a$, and the minimum propagation losses were found to be equal to $1242 \mathrm{~dB} / \mathrm{cm}$. Figures 5(c) and 5(d) show the components of the guided modes' displacement field for this structure at $z=0$. Similarly to design $\mathrm{A}$, the modes remain mostly along the interface, however, the edge state confinement is significantly worse in this case.

We note that Barik et al. have obtained experimental loss measurements on this structure and extracted some values for the optimum loss length [48]. Using their lattice constant of $a=445 \mathrm{~nm}$, our normalized loss length of $L_{\alpha}=79 a$ is equivalent to $L_{\alpha}=35 \mu \mathrm{m}$. Comparing this loss length with their experimental value of $22 \mu \mathrm{m}$, it is clear that these two values are in reasonable agreement, especially as we have not accounted for any other source of loss, and we have extracted 
(a)

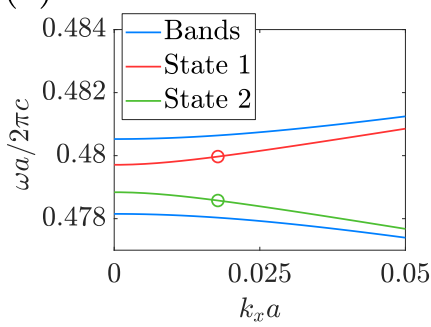

(c)

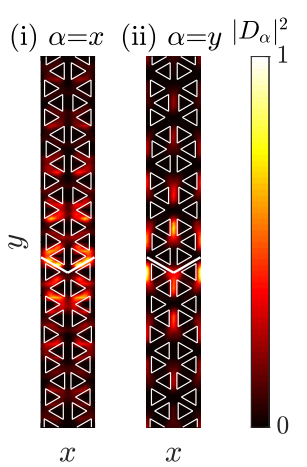

(b)

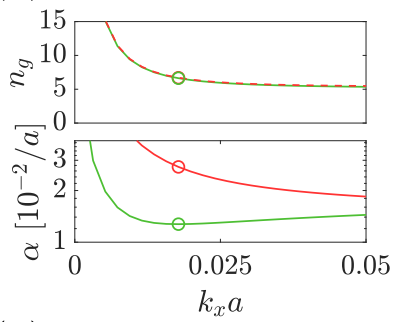

(d)

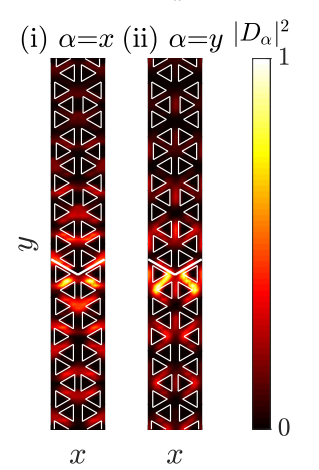

FIG. 5. (a) Zoom-in to design B's guided bands of interest, labeled state 1 and state 2 . Points of interest, which are found above the light line, provide the minimum loss coefficient for state 2 and are indicated by circles. (b) The group index (top), $n_{g}$, and loss coefficient (bottom), $\alpha$, of state 1 and state 2. The two points of interest are represented by circles. (c) Electric displacement field mode profiles of the $x$ and $y$ components of state 1 at the point of interest. (d) Electric displacement field mode profiles of state 2.

the theoretical lowest loss as a limit. We also expect fabrication imperfections to impact these numbers further.

While both design A and design B produce edge state modes that appear to be intrinsically lossy, we stress that the physics of these topological structures is much richer than that of regular PC modes [40,41], and these loss lengths are certainly large enough to probe many finite-size waveguide effects, and for exploiting topology-dependent spin [48].

\section{B. Valley Hall edge state structures of Shalaev et al. [58] and He et al. [59]}

Next, we examine the recent PCS edge state structure of Shalaev et al. [58], described earlier. The GME computations for this structure use a slab dielectric constant of $\epsilon_{s}=12.11$, a slab thickness of $d=0.639 a$, equilateral triangular hole lengths of $L_{1}=0.4 a$ and $L_{2}=0.6 a$, and a lattice constant of $a=423 \mathrm{~nm}$.

The full photonic band diagram, as well as the lattice design, for this topological structure are shown in Fig. 6. Unlike designs $\mathrm{A}$ and $\mathrm{B}$, its interface does not separate two honeycomb lattice structures of expanded and shrunken clusters. Instead, a standard honeycomb lattice of triangular holes with two alternating hole sizes has inversion symmetry applied, and an interface is formed from the larger triangular holes. As a consequence of the periodic nature of the GME in both the $x$ and the $y$ directions, an intermediate interface of smaller triangular holes forms beyond what is shown in Fig. 6. The mode confined within the real interface of larger holes is

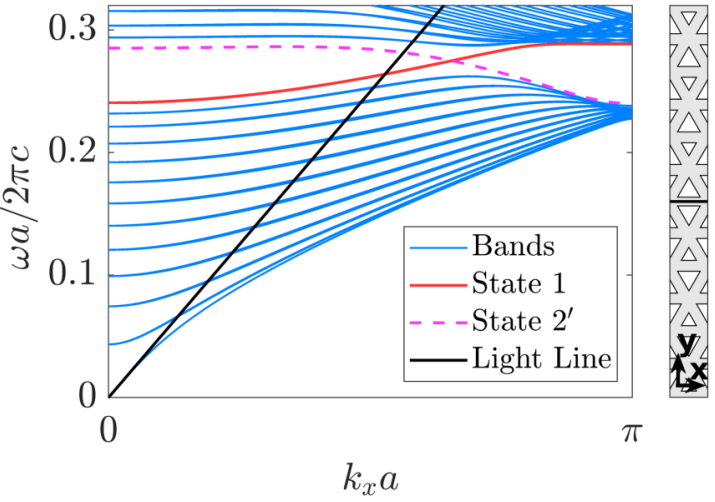

FIG. 6. Dispersion of the edge state structure of triangular holes, design $C$ [58]. Propagation is along $x$.

labeled state 1 , whereas the artificial mode confined to the intermediate interface of smaller holes is labeled state $2^{\prime}$; see Appendix B for more information on how these artificial modes (with respect to the original lattice) are defined and obtained.

Another difference between this structure and designs A and $\mathrm{B}$ is that the guided modes lie below the light line, as shown in the zoom-in of the band diagram in Fig. 7(a).
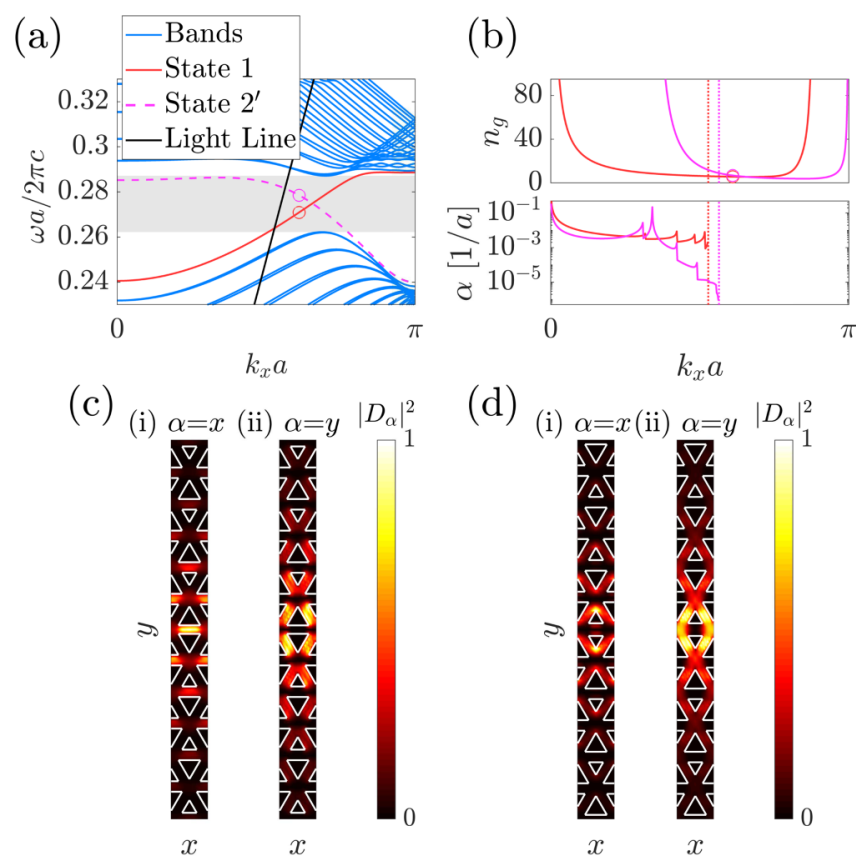

(d)

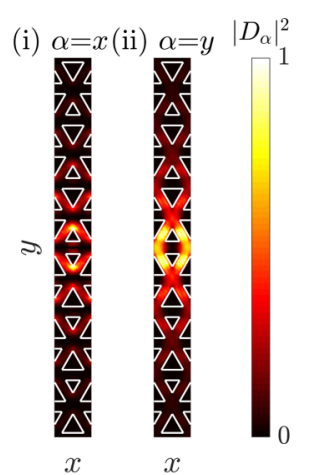

FIG. 7. (a) Zoom-in to design C's guided bands of interest, labeled state 1 and state $2^{\prime}$. Note that state $2^{\prime}$ is in fact an edge state mode at the border of the supercell but constitutes an alternative design. Points of interest, indicated by circles, were chosen to be below the light line. (b) The group index (top), $n_{g}$, and loss coefficient (bottom), $\alpha$, of state 1 and state $2^{\prime}$. The two points of interest are represented by circles, and the light line crossings are indicated by dotted vertical lines. (c) Electric displacement field mode profiles of the $x$ and $y$ components for state 1 at the point of interest. (d) Electric displacement field mode profiles of state $2^{\prime}$; for simplicity, we show the edge state at the center and rearrange the lattice accordingly (see Appendix B). 


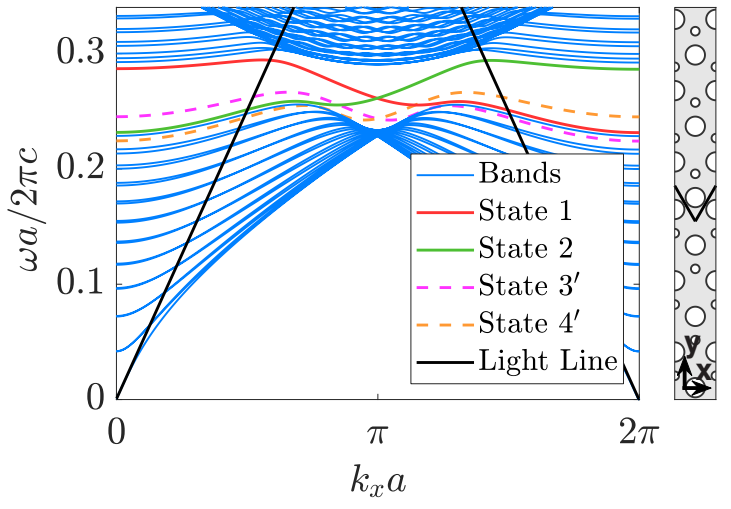

FIG. 8. Dispersion of the edge state structure of circular holes, design D [59]. Propagation is along $x$.

Figure 7(b) shows the corresponding group index, $n_{g}$, and loss coefficient, $\alpha$, for these two modes. The point at which a mode crosses the light line is indicated by a dotted vertical line. The mode point of interest in this case is arbitrarily chosen to be below the light line. As expected from Fig. 7(b), the loss coefficient, $\alpha$, becomes 0 when the mode falls below the light line. The various jumps in $\alpha$ versus wave vector arise from crossings between the Bloch mode and the effective slab waveguide modes (see Appendix A). Also, the significant dip in $\alpha$ for state $2^{\prime}$ is a consequence of the decreasing $n_{g}$ and the larger effective mode volume. As an example, when the $\alpha$ values of state 1 and state $2^{\prime}$ are similar, we obtain effective mode volumes of $0.032 \mu \mathrm{m}^{3}$ [or $0.264\left(\lambda / n_{s}\right)^{3}$ ] for state 1 and $0.038 \mu \mathrm{m}^{3}$ [or $0.489\left(\lambda / n_{s}\right)^{3}$ ] for state $2^{\prime}$; however, when $\alpha$ differs by orders of magnitude, the effective mode volumes increase to $0.039 \mu \mathrm{m}^{3}$ [or $0.391\left(\lambda / n_{s}\right)^{3}$ ] for state 1 and $0.051 \mu \mathrm{m}^{3}$ [or $0.650\left(\lambda / n_{s}\right)^{3}$ ] for state $2^{\prime}$, which are comparable to W1-like effective mode volumes [60].

Figures 7(c) and 7(d) show the components of the displacement field for both the real and the artificial modes of this structure at $z=0$. Unlike designs A and B, this lattice structure shows a significant improvement in terms of waveguide confinement; the edge state modes remain tightly confined to the interface in this case due to having zero losses. For the local chiral properties of these modes, see Appendix C.

Finally, we study another valley Hall edge state structure of $\mathrm{He}$ et al. [59]. For this design, the GME computations use a slab dielectric constant of $\epsilon_{s}=12.04$, a slab thickness of $d=0.571 a$, hole radii of $r_{1}=0.105 a$ and $r_{2}=0.235 a$, and a lattice constant of $a=385 \mathrm{~nm}$. This topological PC design is quite similar to design C; a standard honeycomb lattice of two alternating circular hole sizes has inversion symmetry applied to it, resulting in an interface formed by the larger circular holes. Figure 8 displays this lattice structure, as well as the full photonic band diagram. This structure has four significant edge state modes: two real modes, labeled state 1 and state 2, and two artificial modes, labeled states $3^{\prime}$ and $4^{\prime}$. Similarly to the artificial mode from design $\mathrm{C}$, states $3^{\prime}$ and $4^{\prime}$ arise from an intermediate interface formed beyond the supercell length (see Appendix B).

Another similarity to design $\mathrm{C}$ is the fact that these edge state modes fall below the light line, as shown in the zoom-in in Fig. 9(a). For states 1 and 2, the group index, $n_{g}$, and loss
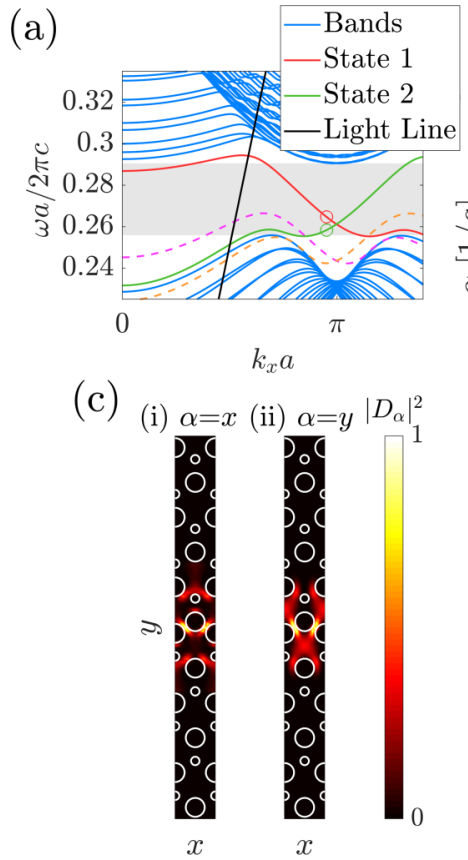

(b)

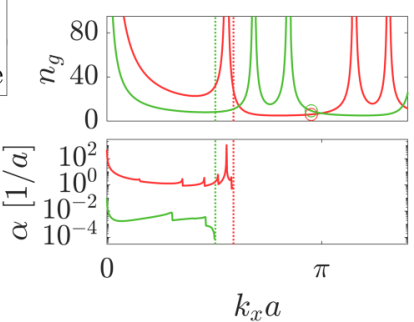

(d)

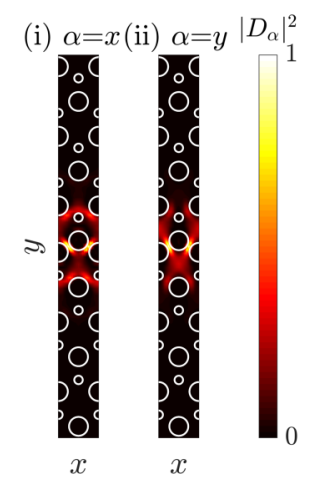

FIG. 9. (a) Zoom-in to design D's guided bands of interest, labeled state 1 and state 2 . States $3^{\prime}$ and $4^{\prime}$, formed at the border of the supercell, are indicated as dashed bands. Points of interest, indicated by circles, were chosen to be below the light line. (b) The group index (top), $n_{g}$, and loss coefficient (bottom), $\alpha$, of state 1 and state 2 . The two points of interest are represented by circles, and the light line crossings are indicated by dotted vertical lines. (c) Electric displacement field mode profiles of the $x$ and $y$ components of state 1 at the point of interest. (d) Electric displacement field mode profiles of state 2 .

coefficient, $\alpha$, are shown in Fig. 9(b); the losses for states $3^{\prime}$ and $4^{\prime}$ are omitted in this case, as the results for states 1 and 2 are more meaningful when compared to the original waveguide design. Once again, the regions below the light line for this structure provide zero losses, and the jumps in $\alpha$ are due to crossings with the effective slab waveguide (see Appendix A). For example effective mode volumes of these modes, we obtain $0.009 \mu \mathrm{m}^{3}$ [or $0.119\left(\lambda / n_{s}\right)^{3}$ ] for state 1 and $0.008 \mu \mathrm{m}^{3}$ [or $0.099\left(\lambda / n_{s}\right)^{3}$ ] for state 2 , which are significantly smaller than those for design $\mathrm{C}$. We also show the components of the displacement field for the two main modes of this structure at $z=0$ in Figs. 9(c) and 9(d). Regions of slow light, small losses, and small effective mode volumes have applications for on-chip quantum light sources including single-photon emitters [60]. Similarly to design C, this structure's edge state modes are very tightly confined to the interface.

\section{CONCLUSIONS}

In this work, we have applied the GME method to study four topological PCS structures, all of which are modifications of the standard honeycomb lattice structure. Two of these structures, designs A and B, proposed by Anderson and Subramania [40] and Barik et al. [41], consist of armchair edge states, with an interface separating shrunken and expanded honeycomb clusters. The edge states of these 
two PCS structures have been shown to fall above the light line, and neither structure seemed to perform particularly well in terms of minimizing propagation loss. Taking previously reported minimum losses of 15 and $5 \mathrm{~dB} / \mathrm{cm}$ for the W1 waveguide for comparison [27,28], these two topological structures show minimum losses of the order of $10^{2}$ and $10^{3} \mathrm{~dB} / \mathrm{cm}$ inside the photonic bandgap. The electromagnetic fields of the guided modes remain mostly along the structures' interfaces, however, these edge states could be more tightly confined.

The two other structures that we have analyzed, designs C and D, proposed by Shalaev et al. [58] and He et al. [59], are valley Hall edge state designs that use inversion symmetry to form an interface. The edge states of both PCS structures fall below the light line, thus providing regions of zero intrinsic losses (neglecting imperfections). The electromagnetic field mode profiles confirm that these edge states are indeed tightly confined to their respective structure's interface. Compared to designs $\mathrm{A}$ and $\mathrm{B}$, these valley Hall edge state designs seem far superior as a result of their good bandwidth and lossless propagation modes. However, it remains to be quantified how these structures are affected by structural disorder, a topic we will explore in future work.

All of the presented edge state modes show interesting chiral features for the Bloch mode polarization, which is useful for coupling to spin-charged quantum dots and realizing unidirectional propagation [61,62]. Further information on the chiral features of the Bloch modes is presented in Appendix C.

\section{ACKNOWLEDGMENTS}

This work was supported by the Natural Sciences and Engineering Research Council of Canada, Queen's University, and the Canadian Foundation for Innovation.

\section{APPENDIX A: FURTHER DETAILS ON THE PROPAGATION LOSS FEATURES}

In Figs. 7(b) and 9(b), we see several peaks and nontrivial features in the loss coefficient $\alpha$ as a function of the wave vector. Apart from the influence of the group index $n_{g}$, these effects can be explained by considering the effective slab waveguide, formed by the effective dielectric constant $\bar{\epsilon}$ of the PCS structure's core layer. The guided modes of this effective slab are folded when considering the Bloch boundary condition, and the PCS modes will inevitably cross with these guided modes. When these bands cross, the effective slab's guided modes act as a loss channel, thus briefly increasing the losses of the PCS's modes, represented by the frequency's imaginary component $\operatorname{Im}(\omega)$. These peaks are more likely to appear for relatively thicker slabs or those with low contrast in the refractive index.

Figures 10(a) and 10(b) demonstrate the origin of this phenomenon for designs C and D of Shalaev et al. [58] and He et al. [59], respectively. The two states of interest of these two topological PCS structures are overlaid onto the guided modes of the effective slab to show the locations where the bands intersect, which correspond to the locations of the jumps in $\alpha$. Note that the bands shown in this case are only TE-like
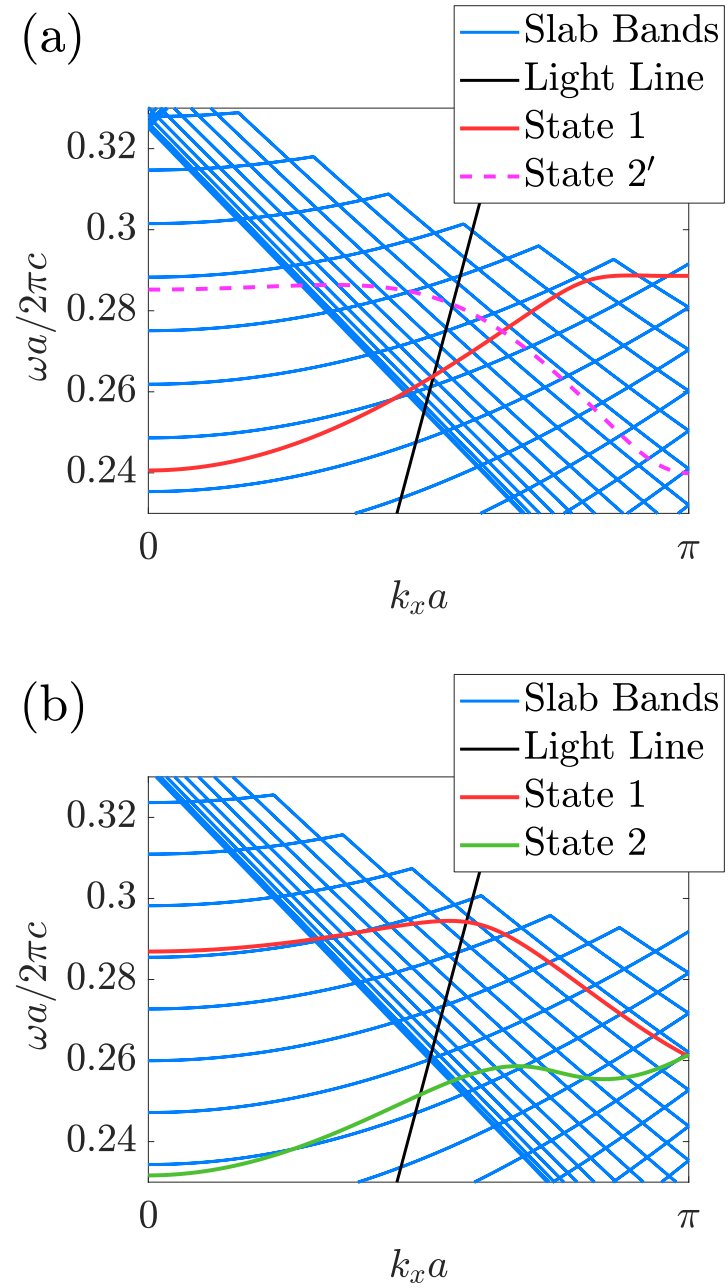

FIG. 10. Dispersion of the effective slabs of the edge state structures of (a) Shalaev et al. [58] and (b) He et al. [59], designs C and $\mathrm{D}$ in the text, respectively. The two waveguide modes of interest for both structures are overlaid onto this photonic band diagram to show the various instances of band crossings. These crossings result in peaks in $\operatorname{Im}(\omega)$ due to the effective guided slab modes acting as loss channels.

modes. The other factors that influence the loss calculations include the group index, $n_{g}$, and the strength of the normalized Bloch modes (or the effective mode volume [60]); larger $n_{g}$ and smaller effective mode volumes both increase the loss in the golden rule.

\section{APPENDIX B: ADDITIONAL MODE PLOTS AND EDGE STATE MODES IDENTIFIED AT THE EDGE OF THE GUIDED-MODE EXPANSION SUPERCELL}

The GME, being periodic in nature, presents a specified boundary condition when building two-dimensional lattice structures. Unlike methods that can apply open boundary conditions, such as the FDTD using perfectly matched layers, the GME builds a lattice structure from an initial unit cell that is repeated periodically in two dimensions. For regular nonwaveguide structures, a lattice can be easily built from its clearly defined unit cell. However, waveguidelike structures require a much larger supercell, which is in general a 
(a)

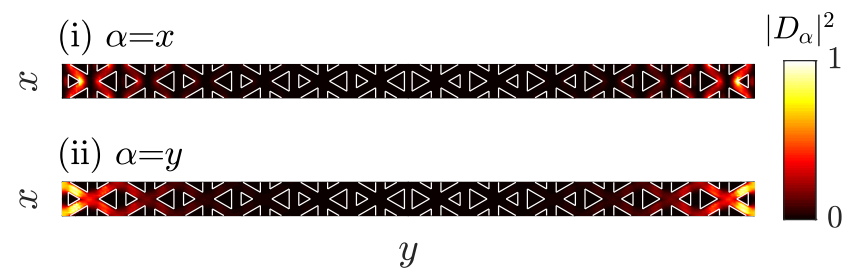

(b)

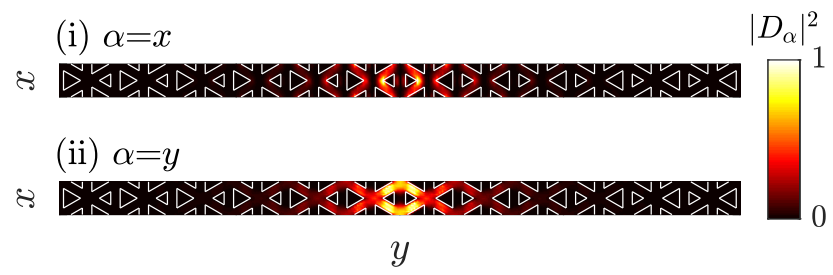

FIG. 11. Electric displacement field mode profiles for state $2^{\prime}$ of the edge state structure of Shalaev et al. [58] (design C in the text). (a) The full supercell of the real design is shown, demonstrating that state $2^{\prime}$ lies at the border of the supercell at an intermediate interface. (b) The lattice structure is altered such that the intermediate interface is at the center of the supercell. State $2^{\prime}$ remains confined at this interface nonetheless.

rectangular section of the lattice with an interface located at the center. Unlike traditional unit cells, these supercells are only repeated periodically in one dimension, which we define as $x$. The $y$ dimension represents the supercell's length, which is assumed to be infinitely long.

Despite these supercells only being periodic in $x$, the GME still views them as being periodic in both $x$ and $y$. Therefore, a supercell length defined as $l_{y}$ will form interfaces at $y= \pm n l_{y}$, where $n=\{0,1,2, \ldots\}$. In principle, we must ensure that $l_{y}$ is large enough such that the neighboring interfaces at $y= \pm l_{y}$ do not interfere with the GME computations. For designs A and $\mathrm{B}$, these periodic interfaces are formed easily, as they are constructed from typical armchair interfaces. Designs C and $\mathrm{D}$, however, utilize inversion symmetry to form an interface, and thus to achieve a perfectly periodic supercell in the $y$ direction, inversion symmetry must be applied twice. Doing so results in intermediate interfaces located at $\pm(n+1 / 2) l_{y}$. The calculations are still physical in the sense that the results have converged, and if we increased the size of the supercell, we could get the same answer. Thus, the GME can find other designs from the boundary of the supercell.

Due to the formation of these intermediate interfaces, new modes are formed. These modes, although fictitious from the input design, can be viewed as a real solution to a new design where the intermediate interface is located at $y=0$. One such artificial mode is shown in design $C$ in the text, labeled state $2^{\prime}$. Figure 11 shows the full supercell of the electric displacement field modes of state $2^{\prime}$ using two different approaches. In Fig. 11(a), the real lattice design is utilized to demonstrate that the confinement occurs at the edge of the supercell at $y= \pm l_{y} / 2$, where the intermediate interface is located. An alternative view of this mode is shown in Fig. 11(b), where the intermediate interface is placed at $y=0$. These two modes are (a)

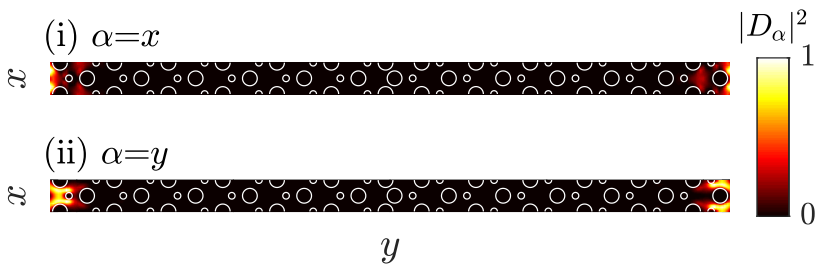

(b)

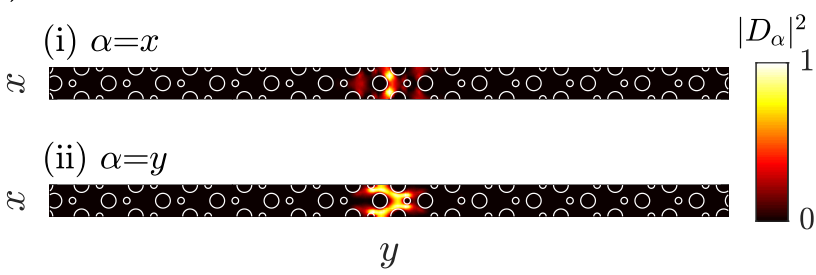

(c)

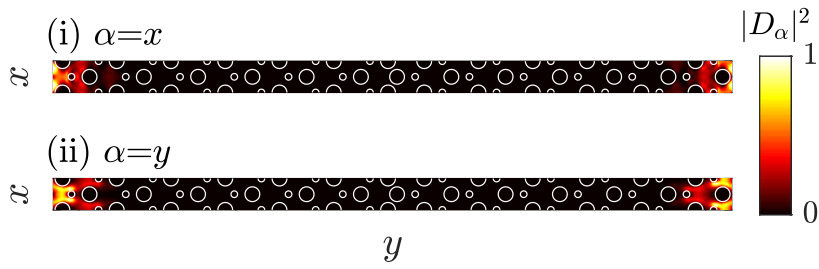

(d)

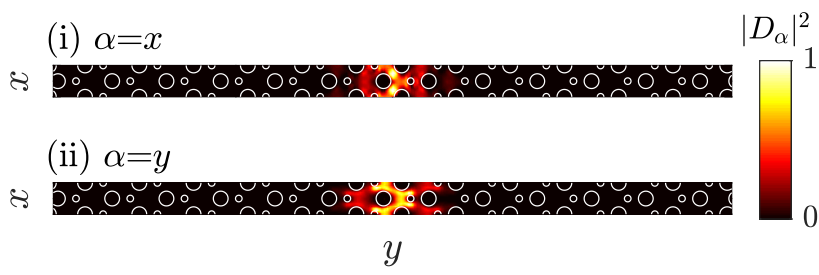

FIG. 12. Electric displacement field mode profiles for states 3 and $4^{\prime}$ of the edge state structure of He et al. [59] (design D in the text). (a) The full supercell of the real design is shown, demonstrating that state $3^{\prime}$ lies at the border of the supercell at an intermediate interface. (b) The lattice structure is altered such that the intermediate interface is at the center of the supercell. State $3^{\prime}$ remains confined at this interface nonetheless. (c) The full supercell of the real design is shown, demonstrating that state $4^{\prime}$ lies at the border of the supercell at an intermediate interface. (d) The lattice structure is altered such that the intermediate interface is at the center of the supercell. State $4^{\prime}$ remains confined at this interface nonetheless.

effectively identical, save a translation of $l_{y} / 2$ applied in the $y$ direction.

The same phenomenon is found in design $\mathrm{D}$ in the text, albeit with two artificial modes rather than one. Figures 12(a) and 12(b) show the electric displacement field mode profiles of state $3^{\prime}$ and Figs. 12(c) and 12(d) show those of state $4^{\prime}$. Once again, the full supercells are shown in these figures to emphasize the artifical states being located at the edges of the supercells. We show once more alternative views of these modes by wrapping the edges to the center, which can be seen as entirely new topological PCS designs. 

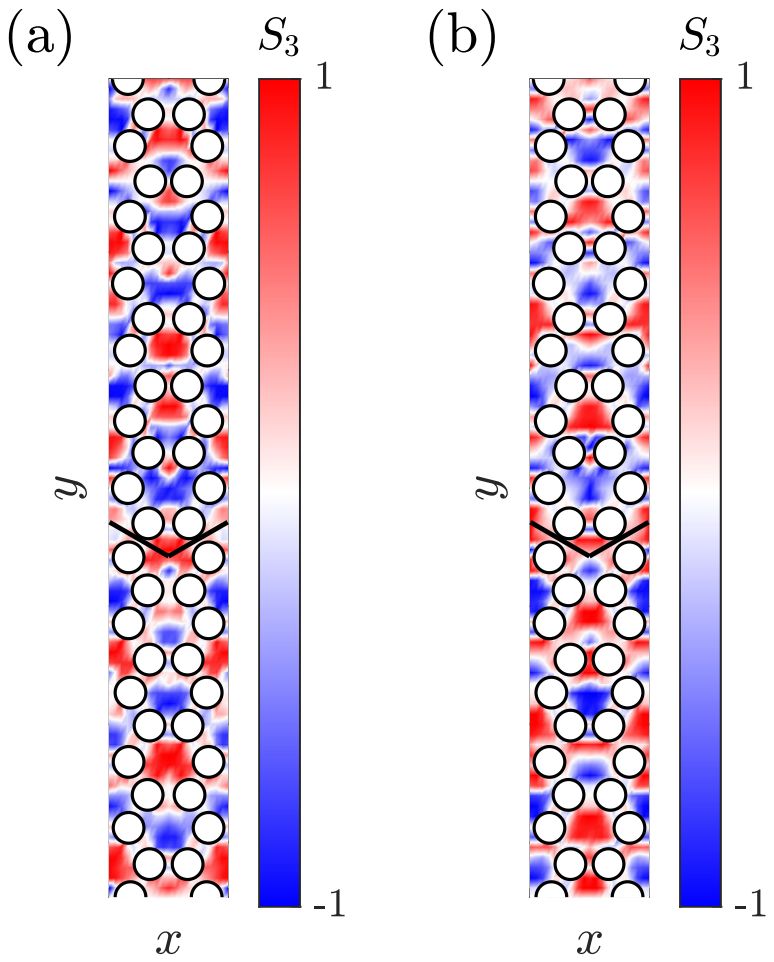

FIG. 13. Polarization for design A's (a) state 1 and (b) state 2, represented by the $S_{3}$ Stokes' parameter.

\section{APPENDIX C: CHIRAL FEATURES OF THE BLOCH MODES}

Here, we display the chiral features of the Bloch modes shown in the text to better highlight the regions of circular po-
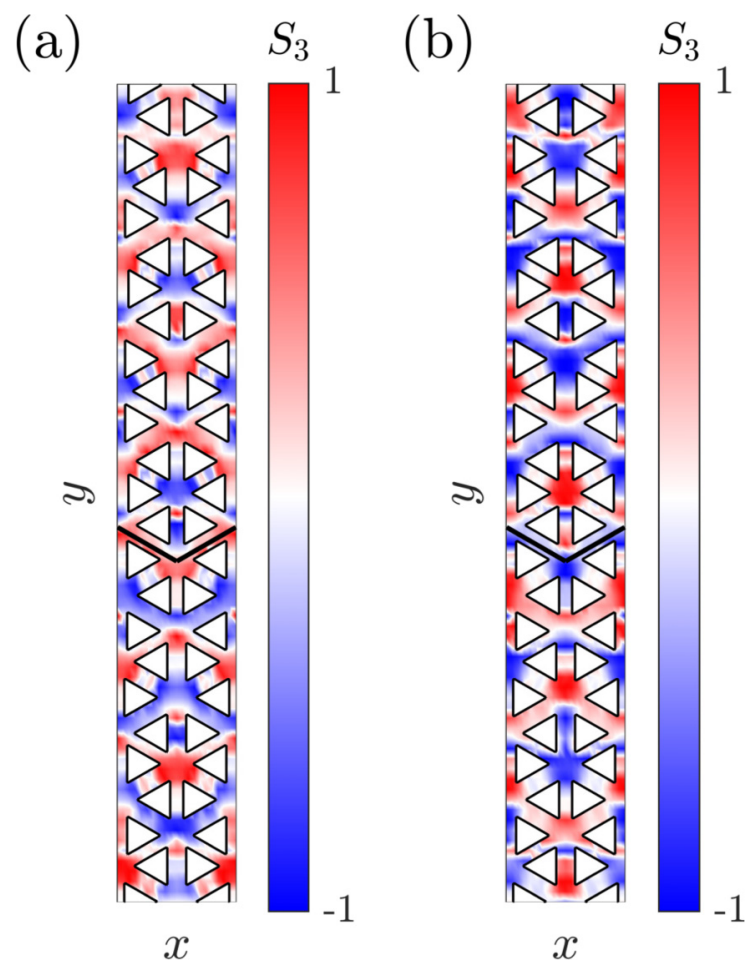

FIG. 14. Polarization for design B's (a) state 1 and (b) state 2, represented by the $S_{3}$ Stokes' parameter.
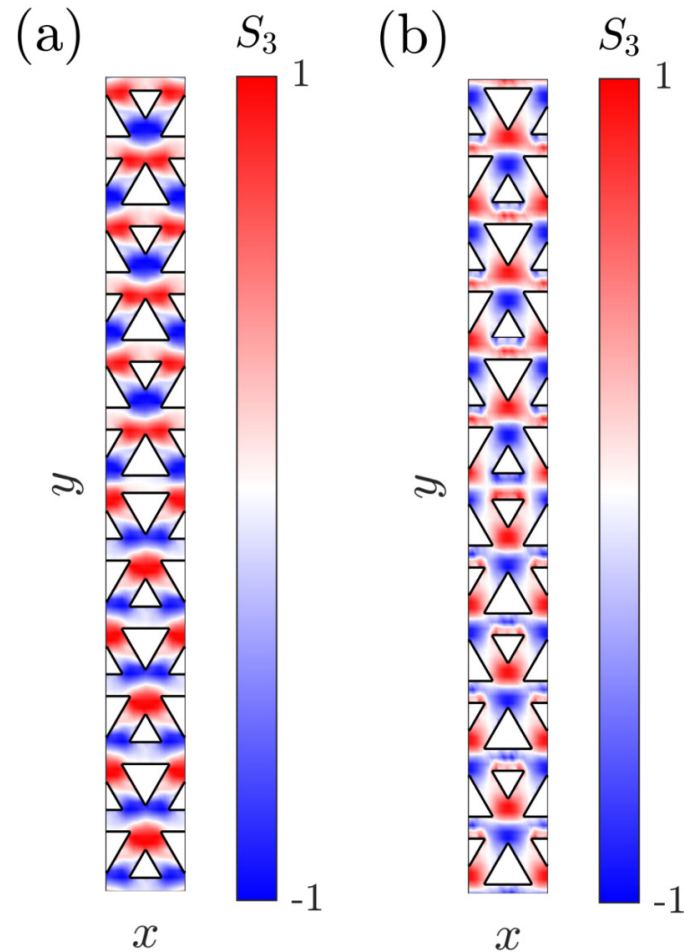

FIG. 15. Polarization for design C's (a) state 1 and (b) state $2^{\prime}$, represented by the $S_{3}$ Stokes' parameter.

larization, which can be used to couple to spin-charged dipole emitters $[48,63]$ and form one-way edge states. Although not necessary for comprehension of the text, understanding the chiral properties of these structures has significant value in terms of quantum applications [62,64]. The method used to show the chiral features of the text's four structures is through the Stokes' parameters $S_{0,1,2,3}$ as a function of position $\boldsymbol{r}$, which describe the polarization of the electric field $\boldsymbol{E}(\boldsymbol{r})$ :

$$
\begin{aligned}
& S_{0}(\boldsymbol{r})=\left|E_{x}(\boldsymbol{r})\right|^{2}+\left|E_{y}(\boldsymbol{r})\right|^{2}, \\
& S_{1}(\boldsymbol{r})=\left(\left|E_{x}(\boldsymbol{r})\right|^{2}-\left|E_{y}(\boldsymbol{r})\right|^{2}\right) / S_{0}(\boldsymbol{r}), \\
& S_{2}(\boldsymbol{r})=2 \operatorname{Re}\left[E_{x}^{*}(\boldsymbol{r}) E_{y}(\boldsymbol{r})\right] / S_{0}(\boldsymbol{r}), \\
& S_{3}(\boldsymbol{r})=2 \operatorname{Im}\left[E_{x}^{*}(\boldsymbol{r}) E_{y}(\boldsymbol{r})\right] / S_{0}(\boldsymbol{r}) .
\end{aligned}
$$

The $z$ component of the Bloch mode fields is negligible, and we show mode profiles below at the slab center only, namely, at $z=0$.

The main benefit of the Stokes' parameters is that they can easily pinpoint the locations of polarization singularities, such as circular polarization ("C points") and linear polarization ("L lines") [62]. The first parameter, $S_{0}$, represents the total electric field strength, which is found from its $x$ and $y$ components. The other three Stokes' parameters, $-1 \leqslant S_{1,2,3} \leqslant 1$, are the three Cartesian positions of the Poincaré sphere [64]. The parameter of interest here is $S_{3}$, due to its feature of being able to easily identify $\mathrm{C}$ points and $\mathrm{L}$ lines. Points where $S_{3}= \pm 1$ represent C points with left and right circular polarization, respectively; similarly, points where $S_{3}=0$ depict L lines. 


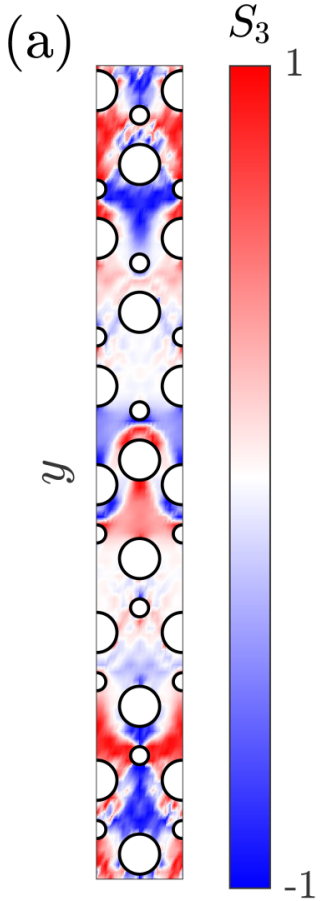

$x$

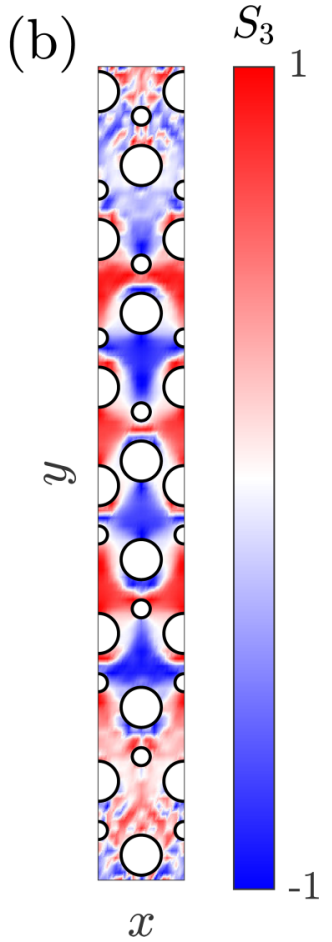

FIG. 16. Polarization for design D's (a) state 1 and (b) state 2, represented by the $S_{3}$ Stokes' parameter.
To highlight the main chiral features, we show the text's four structures in terms of $S_{3}$. Doing so is quite trivial, as the $x$ and $y$ components of the electric displacement field $\boldsymbol{D}$ are available to us. Using the relation $\boldsymbol{D} \equiv \epsilon \boldsymbol{E}$, the Stokes' parameters are easily found for each structure's supercell. The values of $S_{3}(\boldsymbol{r})$ for the eight states of interest in the text are shown in Figs. 13-16, featuring designs A-D, respectively.

The polarizations of the lossy armchair PCS structures (designs A and B) are very much alike due to their similar armchair configurations. We can see that these structures tend to preserve both left and right circularly polarized $\mathrm{C}$ points within the honeycomb clusters. There do not seem to be any other discernible features for these two structures other than the scarcity of L lines.

For design $\mathrm{C}$, there is a much more even distribution of $\mathrm{C}$ points than for the previous two structures. However, due to having more locations with $\mathrm{L}$ lines, this structure seems to be more versatile. State 1 and state $2^{\prime}$ are quite similar, yet state 1 's $C$ points are slightly more defined than those of state 2 '.

Design D's chiral features are similar to those of designs A and $\mathrm{B}$ in the sense that the $\mathrm{C}$ points tend to cluster, however, they do so around the holes. Additionally, like design C, there are significantly more $\mathrm{L}$ lines in this case. It is also easier to identify the interface of this structure, as the $\mathrm{C}$ points tend to gather around it. This is consistent with results found by He et al., which demonstrate that left and right circularly polarized $\mathrm{C}$ points are found at the center of the interface's holes [59].
[1] K. Sakoda, Optical Properties of Photonic Crystals, 2nd ed. Springer Series in Optical Sciences (Springer-Verlag, Berlin, 2005).

[2] M. Patterson, S. Hughes, S. Schulz, D. M. Beggs, T. P. White, L. O'Faolain, and T. F. Krauss, Disorder-induced incoherent scattering losses in photonic crystal waveguides: Bloch mode reshaping, multiple scattering, and breakdown of the BeerLambert law, Phys. Rev. B 80, 195305 (2009).

[3] M. Patterson, Classical and quantum optical properties of slow light photonic crystal waveguides, M.Sc. thesis, Queen's University, Canada, 2009.

[4] N. Mann, Theoretical and computational studies of disorderinduced scattering and nonlinear optical interactions in slowlight photonic crystal waveguides, Ph.D. thesis, Queen's University, Canada, 2017.

[5] T. F. Krauss, Why do we need slow light? Nat. Photon. 2, 448 (2008).

[6] M. Notomi, K. Yamada, A. Shinya, J. Takahashi, C. Takahashi, and I. Yokohama, Extremely Large Group-Velocity Dispersion of Line-Defect Waveguides in Photonic Crystal Slabs, Phys. Rev. Lett. 87, 253902 (2001).

[7] Y. A. Vlasov, M. O'Boyle, H. F. Hamann, and S. J. McNab, Active control of slow light on a chip with photonic crystal waveguides, Nature 438, 65 (2005).

[8] T. Baba, Slow light in photonic crystals, Nat. Photon. 2, 465 (2008).

[9] M. Minkov and V. Savona, Wide-band slow light in compact photonic crystal coupled-cavity waveguides, Optica 2, 631 (2015).
[10] N. Matsuda, T. Kato, K. Harada, H. Takesue, E. Kuramochi, H. Taniyama, and M. Notomi, Slow light enhanced optical nonlinearity in a silicon photonic crystal coupled-resonator optical waveguide, Opt. Express 19, 19861 (2011).

[11] Y. Zhang and B. Li, Photonic crystal-based bending waveguides for optical interconnections, Opt. Express 14, 5723 (2006).

[12] Y. Akahane, T. Asano, B.-S. Song, and S. Noda, High-Q photonic nanocavity in a two-dimensional photonic crystal, Nature 425, 944 (2003).

[13] B.-S. Song, S. Noda, T. Asano, and Y. Akahane, Ultra-high-Q photonic double-heterostructure nanocavity, Nat. Mater. 4, 207 (2005).

[14] M. Minkov, V. Savona, and D. Gerace, Photonic crystal slab cavity simultaneously optimized for ultra-high $\mathrm{Q} / \mathrm{V}$ and vertical radiation coupling, Appl. Phys. Lett. 111, 131104 (2017).

[15] T. Asano, Y. Ochi, Y. Takahashi, K. Kishimoto, and S. Noda, Photonic crystal nanocavity with a $\mathrm{Q}$ factor exceeding eleven million, Opt. Express 25, 1769 (2017).

[16] K. Hennessy, A. Badolato, M. Winger, D. Gerace, M. Atatüre, S. Gulde, S. Fält, E. L. Hu, and A. Imamoğlu, Quantum nature of a strongly coupled single quantum dot-cavity system, Nature 445, 896 (2007).

[17] T. Yoshie, A. Scherer, J. Hendrickson, G. Khitrova, H. M. Gibbs, G. Rupper, C. Ell, O. B. Shchekin, and D. G. Deppe, Vacuum Rabi splitting with a single quantum dot in a photonic crystal nanocavity, Nature 432, 200 (2004).

[18] B. Ellis, M. A. Mayer, G. Shambat, T. Sarmiento, J. Harris, E. E. Haller, and J. Vučković, Ultralow-threshold electrically pumped 
quantum-dot photonic-crystal nanocavity laser, Nat. Photon. 5, 297 (2011).

[19] K. Nozaki, T. Tanabe, A. Shinya, S. Matsuo, T. Sato, H. Taniyama, and M. Notomi, Sub-femtojoule all-optical switching using a photonic-crystal nanocavity, Nat. Photon. 4, 477 (2010).

[20] U. P. Dharanipathy, M. Minkov, M. Tonin, V. Savona, and R. Houdré, High-Q silicon photonic crystal cavity for enhanced optical nonlinearities, Appl. Phys. Lett. 105, 101101 (2014).

[21] M. Notomi, E. Kuramochi, and H. Taniyama, Ultrahigh-Q nanocavity with 1D photonic gap, Opt. Express 16, 11095 (2008).

[22] Y. Tanaka, T. Asano, and S. Noda, Design of photonic crystal nanocavity with $Q$-factor of $\sim 10^{9}$, J. Lightwave Technol. 26, 1532 (2008).

[23] J. P. Vasco, H. Vinck-Posada, P. T. Valentim, and P. S. S. Guimãraes, Modeling of Fano resonances in the reflectivity of photonic crystal cavities with finite spot size excitation, Opt. Express 21, 31336 (2013).

[24] K. Kitano, K. Suzuki, K. Ishizaki, and S. Noda, Three-dimensional photonic crystals fabricated by simultaneous multidirectional etching, Phys. Rev. B 91, 155308 (2015).

[25] T. Y. M. Chan, O. Toader, and S. John, Photonic band-gap formation by optical-phase-mask lithography, Phys. Rev. E 73, 046610 (2006).

[26] M. Patterson, S. Hughes, S. Combrié, N.-V.-Q. Tran, A. De Rossi, R. Gabet, and Y. Jaouën, Disorder-Induced Coherent Scattering in Slow-Light Photonic Crystal Waveguides, Phys. Rev. Lett. 102, 253903 (2009).

[27] E. Kuramochi, M. Notomi, S. Hughes, A. Shinya, T. Watanabe, and L. Ramunno, Disorder-induced scattering loss of linedefect waveguides in photonic crystal slabs, Phys. Rev. B 72, 161318(R) (2005).

[28] L. O'Faolain, T. P. White, D. O'Brien, X. Yuan, M. D. Settle, and T. F. Krauss, Dependence of extrinsic loss on group velocity in photonic crystal waveguides, Opt. Express 15, 13129 (2007).

[29] J. Li, T. P. White, L. O'Faolain, A. Gomez-Iglesias, and T. F. Krauss, Systematic design of flat band slow light in photonic crystal waveguides, Opt. Express 16, 6227 (2008).

[30] N. Mann, S. Combrié, P. Colman, M. Patterson, A. D. Rossi, and S. Hughes, Reducing disorder-induced losses for slow light photonic crystal waveguides through Bloch mode engineering, Opt. Lett. 38, 4244 (2013).

[31] N. Mann, M. Patterson, and S. Hughes, Role of Bloch mode reshaping and disorder correlation length on scattering losses in slow-light photonic crystal waveguides, Phys. Rev. B 91, 245151 (2015).

[32] S. Hughes, L. Ramunno, J. F. Young, and J. E. Sipe, Extrinsic Optical Scattering Loss in Photonic Crystal Waveguides: Role of Fabrication Disorder and Photon Group Velocity, Phys. Rev. Lett. 94, 033903 (2005).

[33] D. Gerace and L. C. Andreani, Effects of disorder on propagation losses and cavity q-factors in photonic crystal slabs, Photon. Nanostruct. Fund. Appl. 3, 120 (2005).

[34] N. L. Thomas and R. Houdré, Group velocity and energy transport velocity near the band edge of a disordered coupled cavity waveguide: An analytical approach, J. Opt. Soc. Am. B 27, 2095 (2010).
[35] M. S. Mohamed, Y. Lai, M. Minkov, V. Savona, A. Badolato, and R. Houdré, Influence of disorder and finite-size effects on slow light transport in extended photonic crystal coupled-cavity waveguides, ACS Photon. 5, 4846 (2018).

[36] D. Gerace and L. C. Andreani, Disorder-induced losses in photonic crystal waveguides with line defects, Opt. Lett. 29, 1897 (2004).

[37] W. Song, R. A. Integlia, and W. Jiang, Slow light loss due to roughness in photonic crystal waveguides: An analytic approach, Phys. Rev. B 82, 235306 (2010).

[38] M. Minkov and V. Savona, Effect of hole-shape irregularities on photonic crystal waveguides, Opt. Lett. 37, 3108 (2012).

[39] J. P. Vasco and S. Hughes, Exploiting long-range disorder in slow-light photonic crystal waveguides: Anderson localization and ultrahigh Q/V cavities, ACS Photon. 6, 2926 (2019).

[40] P. D. Anderson and G. Subramania, Unidirectional edge states in topological honeycomb-lattice membrane photonic crystals, Opt. Express 25, 23293 (2017).

[41] S. Barik, H. Miyake, W. DeGottardi, E. Waks, and M. Hafezi, Two-dimensionally confined topological edge states in photonic crystals, New J. Phys. 18, 113013 (2016).

[42] N. Parappurath, F. Alpeggiani, L. Kuipers, and E. Verhagen, Direct observation of topological edge states in silicon photonic crystals: Spin, dispersion, and chiral routing, Sci. Adv. 6, eaaw4137 (2020).

[43] L. Lu, J. D. Joannopoulos, and M. Soljačić, Topological photonics, Nat. Photon. 8, 821 (2014).

[44] L. Lu, C. Fang, L. Fu, S. G. Johnson, J. D. Joannopoulos, and M. Soljačić, Symmetry-protected topological photonic crystal in three dimensions, Nat. Phys. 12, 337 (2016).

[45] L.-H. Wu and X. Hu, Scheme for Achieving a Topological Photonic Crystal by Using Dielectric Material, Phys. Rev. Lett. 114, 223901 (2015).

[46] M. J. Mehrabad, A. P. Foster, R. Dost, A. M. Fox, M. S. Skolnick, and L. R. Wilson, Chiral topological photonics with an embedded quantum emitter, arXiv:1912.09943 (2019).

[47] M. B. de Paz, C. Devescovi, G. Giedke, J. J. Saenz, M. G. Vergniory, B. Bradlyn, D. Bercioux, and A. García-Etxarri, Tutorial: Computing topological invariants in 2D photonic crystals, Adv. Quantum Technol. 3, 1900117 (2020).

[48] S. Barik, A. Karasahin, C. Flower, T. Cai, H. Miyake, W. DeGottardi, M. Hafezi, and E. Waks, A topological quantum optics interface, Science 359, 666 (2018).

[49] Y. Zhao, P. Belov, and Y. Hao, Accurate modeling of the optical properties of left-handed media using a finite-difference timedomain method, Phys. Rev. E 75, 037602 (2007).

[50] J. Andreasen, H. Cao, A. Taflove, P. Kumar, and Chang-qi Cao, Finite-difference time-domain simulation of thermal noise in open cavities, Phys. Rev. A 77, 023810 (2008).

[51] N. N. Potravkin, I. A. Perezhogin, and V. A. Makarov, Numerical solution of Maxwell equations by a finite-difference time-domain method in a medium with frequency and spatial dispersion, Phys. Rev. E 86, 056706 (2012).

[52] A. Taflove and S. C. Hagness, Computational Electrodynamics: The Finite-Difference Time-Domain Method (Artech House, 2005), https://us.artechhouse.com/.

[53] H. Kim, J. Park, and B. Lee, Fourier Modal Method and Its Applications in Computational Nanophotonics (CRC Press, Boca Raton, FL, 2012). 
[54] S. G. Johnson and J. D. Joannopoulos, Block-iterative frequency-domain methods for Maxwell's equations in a planewave basis, Opt. Express 8, 173 (2001).

[55] A. Taflove and S. Hagness, Computational Electrodynamics: The Finite-Difference Time-Domain Method, 3rd ed. (Artech House, 2005).

[56] J. D. Joannopoulos (ed.), Photonic Crystals: Molding the Flow of Light, 2nd ed. (Princeton University Press, Princeton, NJ, 2008).

[57] L. C. Andreani and D. Gerace, Photonic-crystal slabs with a triangular lattice of triangular holes investigated using a guidedmode expansion method, Phys. Rev. B 73, 235114 (2006).

[58] M. I. Shalaev, W. Walasik, A. Tsukernik, Y. Xu, and N. M. Litchinitser, Robust topologically protected transport in photonic crystals at telecommunication wavelengths, Nat. Nanotechnol. 14, 31 (2019).

[59] X.-T. He, E.-T. Liang, J.-J. Yuan, H.-Y. Qiu, X.-D. Chen, F.-L. Zhao, and J.-W. Dong, A silicon-on-insulator slab for topological valley transport, Nat. Commun. 10, 872 (2019).
[60] V. S. C. Manga Rao and S. Hughes, Single quantum-dot purcell factor and $\beta$ factor in a photonic crystal waveguide, Phys. Rev. B 75, 205437 (2007).

[61] I. Söllner, S. Mahmoodian, S. L. Hansen, L. Midolo, A. Javadi, G. Kiršanskè, T. Pregnolato, H. El-Ella, E. H. Lee, J. D. Song, S. Stobbe, and P. Lodahl, Deterministic photon-emitter coupling in chiral photonic circuits, Nat. Nanotechnol. 10, 775 (2015).

[62] A. B. Young, A. C. T. Thijssen, D. M. Beggs, P. Androvitsaneas, L. Kuipers, J. G. Rarity, S. Hughes, and R. Oulton, Polarization Engineering in Photonic Crystal Waveguides for Spin-Photon Entanglers, Phys. Rev. Lett. 115, 153901 (2015).

[63] K. M. Ho, C. T. Chan, and C. M. Soukoulis, Existence of a Photonic Gap in Periodic Dielectric Structures, Phys. Rev. Lett. 65, 3152 (1990).

[64] B. Lang, D. M. Beggs, A. B. Young, J. G. Rarity, and R. Oulton, Stability of polarization singularities in disordered photonic crystal waveguides, Phys. Rev. A 92, 063819 (2015). 